RUNNING HEAD: FUTURE DIRECTIONS SINGLE-SESSION INTERVENTIONS

Future Directions in Single-Session Youth Mental Health Interventions

Jessica L. Schleider * a, Mallory L. Dobias †, a, Jenna Y. Sung †a, Michael C. Mullarkey a, b

a Department of Psychology, Stony Brook University

b Department of Psychology, University of Texas at Austin

$\dagger$ Denotes equal co-authorship

* Corresponding author: Jessica L. Schleider, Ph.D., Stony Brook University, Psychology B-340, Stony

Brook, NY 11794-2500; E-mail: jessica.schleider@stonybrook.edu; Tel: (631) 632-4131

CITATION: Schleider, J.L., *Dobias, M.L., *Sung, J.Y., Mullarkey, M.C. (2019, in press). Future directions in single-session youth mental health interventions. Journal of Clinical Child and Adolescent Psychology.

*denotes equal co-authorship 


\begin{abstract}
The United States spends more money on mental health services than any other country, yet access to effective psychological services remains strikingly low. The need-to-access gap is especially wide among children and adolescents, with up to $80 \%$ of youths with mental health needs going without services, and the remainder often receiving insufficient or untested care. Single-session interventions (SSIs) may offer a promising path toward improving accessibility, cost-effectiveness, and completion rates for youth mental health services. SSIs are structured programs that intentionally involve only one visit or encounter with a clinic, provider, or program; they may serve as stand-alone or adjunctive clinical services. A growing body of evidence supports the capacity of SSIs to reduce and prevent youth psychopathology of multiple types. Here, we provide a working definition of SSIs for use in future research and practice; summarize the literature to date on SSIs for child and adolescent mental health; and propose recommendations for the future design, evaluation, and implementation of SSIs across a variety of settings and contexts. We hope that this paper will serve as an actionable research agenda for gauging the full potential of SSIs as a force for youth mental health.
\end{abstract}




\section{Future Directions in Single-Session Youth Mental Health Interventions}

The discrepancy between need and access to mental health services is uncontestable. For children and adolescents, public health consequences of this gap are especially profound. More than half of lifetime mental health problems emerge by age 14 (e.g., de Girolamo, Dagani, Purcell, Cocchi, \& McGorry, 2012; Kessler \& Wang, 2008), often leading to chronic impairments and adverse impacts for individuals, families, and societies. Despite significant strides in the development of psychosocial treatments for youth mental health problems, up to $80 \%$ of youth in the United States with mental health needs receive no services at all (Kataoka, Zhang, \& Wells, 2002; Kessler et al., 2005; Konrad, Ellis, Thomas, Holzer, \& Morrissey, 2009; Kazdin, 2011; Kazdin \& Rabbitt, 2013). Even when services are accessed, the definition of "treatment" is incredibly broad, with evidence-based interventions being the exception, not the rule. More typically, "treatment" involves a single, unstructured contact with a medical doctor, counselor, emergency hotline, religious leader, or other health professional (Kazdin, 2019), the benefits of which are unknown (Chorpita, 2019).

This reality poses a critical challenge to the field of clinical intervention science — one that has been articulated many times before. Eight years ago, Kazdin and Blase (2011) called for a "rebooting" of psychotherapy research and practice, stating that "mental health professionals are not likely to reduce the prevalence, incidence, and burden of mental illness without a major shift in intervention research and clinical practice" via a portfolio of novel approaches to service delivery (Kazdin \& Blase, 2011, p. 21). Soon after, (Kazdin \& Rabbitt, 2013) detailed several models for such delivery approaches, including "best-buy interventions," "good-buy interventions," and "disruptive innovations"—-all paths towards redesigning treatments to extend their reach and population-level impact. Yet again, Kazdin (2019) asked us to reconsider how psychotherapy can and should be delivered: He argues that without removing requirements of a face-to-face format, an 'expert' with years of training, and a physical office, the needto-access gap will likely stay stagnant. Indeed, Kazdin (2019) asserts the need to think about therapeutic action through an entirely new lens, asserting that future interventions may not rely on the psychological treatments that have dominated research to date. 
As Chorpita (2019) aptly noted, the fact that Kazdin and Blase's initial call (2011) is echoed nearperfectly in Kazdin's most recent one (2019) is nothing short of alarming. It highlights the unignorable and overdue need for action towards rebuilding our field's conception of where, how, and by whom effective mental health services can exist. Any action-focused path to reducing the need-to-access gap will require moving beyond the dominant settings, formats, and systems that have constrained intervention delivery to date. Indeed, as Chorpita (2019) asserts, "it may well be time we move past thinking about 'treatments' as our only form of solutions" (p. 475), with "treatments" referencing the dominant design of current psychosocial therapies: weekly, clinician-delivered interventions in brick-andmortar clinical settings, which are too often inaccessible to those they are designed to serve.

In this paper, we will overview one promising approach to accomplishing the goals outlined by Kazdin (2019), Chorpita (2019), and numerous others: Single-Session Interventions (henceforth SSIs). Even among youths who do access services, treatment is often brief: U.S. youths who begin therapy attend an average of just 3.9 sessions (Harpaz-Rotem, Leslie, \& Rosenheck, 2004), and the modal number of sessions attended is one (Hoyt, Bobele, Slive, Young, \& Talmon, 2018). This creates a need to quantify and capitalize on what can be accomplished, given appropriate targeting and structure, in a short period of time. We will first provide a working definition of SSIs and differentiate them from other nontraditional modes of service delivery. Next, we will summarize the literature on SSIs for youth mental health problems to date. We will then outline our team's approach and recommendations for developing and testing SSIs and propose future directions for researchers, practitioners, and policy-makers to gauge and realize their full potential.

Quantifying SSIs' promise creates an opportunity for a paradigm shift in our field's thinking about constructing services for broad-scale impact. SSIs can operate as stand-alone services or as adjunctive supports within existing care systems; as such, the study of SSIs may improve the reach of accessible mental health interventions, helping to mitigate problems linked to long waiting lists, the global provider shortage, and high costs of traditional care (Hoyt et al., 2018; Duvall, Young, \& Kayes- 
Burden, 2012). We hope that this paper will serve as a useful guide for others interested in exploring what SSIs can offer to youths, providers, and systems of care.

\section{Defining Single-Session Interventions}

The concept of planned single-session treatment was first articulated by Talmon (1990, p. xv) in Single Session Therapy: Maximizing the Effect of the First (and Often Only) Therapeutic Encounter: "Single-session therapy is defined...as one face-to-face meeting between a therapist and patient with no previous or subsequent sessions within one year." We conceptualize SSIs through a similar lens, but including alternative delivery models made possible by technological advances (e.g., digital, selfadministered SSIs). We define SSIs as specific, structured programs that intentionally involve just one visit or encounter with a clinic, provider, or program (1-day interventions involving multiple "modules," but occurring in one visit, qualify under this definition; Schleider \& Weisz, 2017). Intentionality in SSIs is critical; we would not consider early dropout from weekly psychotherapy, nor an unstructured, unplanned encounter with a healthcare professional, to constitute an SSI. Moreover, SSIs do not reflect a specific theoretical orientation. They can and have employed a wide diversity of approaches (e.g., solution-focused, cognitive-behavioral, and psychodynamic techniques); the "client" may be an individual youth, a family, or a group; and the session may occur in an office, a hospital, a school, or at home, in the case of digitally-mediated programs. SSIs thus reference the intentional delivery of some singleencounter program, not a specific strategy or orientation.

SSIs reflect a subset of "brief, intensive, and concentrated" (BIC) interventions for youth psychopathology. BIC interventions can vary widely in length, from one-time, 2.5 hour therapies for specific phobia to 10-day, intensive interventions for panic disorder (Öst \& Ollendick, 2017). Given that many BICs can still be costly (considering expenses linked with provider training, session fees, time commitment, and in some cases, travel to specific clinics in the country where such treatments are offered; Ollendick, Ryan, Capriola-Hall, Austin, \& Fraire, 2018), we view SSIs as an especially scalable BIC treatment subtype.

\section{State of the Evidence on Single-Session Interventions for Youth}


Evidence suggests that SSIs can help reduce or prevent psychopathology in youth. Randomized trials have shown promising effects for diverse youth problem types, including specific phobias (Davis, Ollendick, \& Ost, 2012), conduct disorder (Mejia, Calam, \& Sanders, 2015), and general distress in multi-problem youths (Perkins, 2006). In a meta-analysis of 50 randomized-controlled trials (Schleider \& Weisz, 2017), SSIs for youth psychological problems demonstrated a significant beneficial effect, $g=.32$; reflecting a "small-to-medium" overall effect (Cohen, 2013) and a 59\% chance that a randomly-selected youth receiving an SSI would show larger symptom reductions than a randomly-selected youth assigned to a control condition (Ruscio \& Mullen, 2012). This effect size was consistent across different levels of youth problem severity and diagnostic status, suggesting SSIs' capacity to benefit youth with low, moderate, and severe mental health problems. Further, significant SSI effects emerged even for SSIs that were self-administered by youths, in the absence of therapist guidance $(g=.32)$. Numerically, SSIs' overall effects are slightly smaller than those for traditional, multi-session youth psychotherapy (Weisz et al., 2017; mean $g=.46$ for treatments lasting 16 sessions, on average). However, their relative brevity and accessibility suggests potential for larger-scale impact.

Further investigation is needed to determine SSIs' promise and limitations. For instance, SSIs have been most effective in reducing youth anxiety and conduct problems, whereas SSIs targeting youth depression showed nonsignificant overall effects at the time this meta-analysis was conducted (Schleider $\&$ Weisz, 2017). That said, intervention effects on youth depression have been relatively weak even for multi-session therapies (Weisz et al., 2017) and only 6 trials in this meta-analysis targeted depression, ensuring a poorly-powered significance test. Additionally, SSIs' overall effects have tended to wane over time, and a majority of included studies in the meta-analysis used inactive controls. More rigorous, longer-term trials of SSIs targeting adolescents, depression, and associated problems thus emerged as high-priority next-steps at the time of this meta-analysis.

Several new RCTs have been conducted since Schleider \& Weisz's (2017) review, suggesting progress in several domains. To provide an up-to-date view of the SSI literature to date, we conducted a literature search specifying a publication period from January 1, 2016 to August 15, 2019, to identify all 
randomized trials of SSIs conducted in the past 3.5 years (the search end-date of the meta-analysis was December 31, 2015). We used the same search and inclusion criteria as in Schleider \& Weisz's (2017) review. This search yielded 8 randomized trials testing SSIs for youth mental health problems, each of which is summarized below, separated by delivery approach (therapist-administered versus selfadministered) and youth problem type. Effect sizes (Cohen's $d$ ) reflecting changes in mental health outcomes for youths in an SSI condition, relative to a control condition, are reported where calculable. 1

\section{Therapist administered SSIs since 2016}

Depression. Two trials of therapist-administered SSIs targeting adolescent depression were conducted since January 2016. A 50-60 minute, single session intervention targeting implicit theories of personality (the notion that personal traits, specifically aggression and peer victimization, are malleable rather than fixed) demonstrated mixed effects in the school setting; adolescents in 8th grade achieved significantly improved depression symptoms from baseline to 6 months and 12 months when compared to control group ( $d s=0.63$ at 6 month, 0.68 at 12 months), whereas adolescents in 9 th grade showed opposite effects ( $d s=-0.54$ at 6 months, -0.58 at 12 months) (Calvete et al., 2019). In another trial, Ranney and colleagues (2017), tested a therapist-delivered motivational interviewing-based SSI targeting alcohol use and peer violence in adolescents (ages 14-18) referred to an emergency department. Those who received the therapist-administered SSI reported significantly lower depressive symptoms at 3 month follow-up, but not at 6-month or 12-month follow-ups, versus youths who received a list of local resources.

Anxiety. Two trials of SSIs targeting youth anxiety were identified. One trial tested whether a group-based, single session intervention for anxious parents reduced anxiety symptoms in offspring (Cartwright-Hatton et al., 2018). Children with parents in the control group were $16.5 \%$ more likely than those whose parents received the SSI to have anxiety disorders at one-year follow-up; further, parents rated the SSI as highly acceptable. Another SSI focusing on "in situ skills training" (IST) was tested as a

1 In some studies, sample sizes were not reported by intervention condition at follow-up points. Effect sizes were not calculable in these cases. 
standalone intervention and as an adjunct to a 5-week, school-based program promoting risk disclosure among foster care-involved youth (White et al., 2019). The IST-only condition significantly increased children's disclosure intentions, but not symptoms of anxiety $(d=0.11)$, relative to a waiting-list control.

Other. Issner and colleagues (2017) evaluated two SSIs targeting positive health behavior in a primary care setting among low-income, ethnic minority adolescents. Participants received either a 3-5 minute goal-identification session, during which youths identified goals relevant to a perceived difficulty in their diet or physical activity regimen, or a 15-20 minute goal-identification session enhanced with brief motivational interviewing (e.g., identifying barriers to success and ways to address them). Both interventions significantly, but not differentially, increased healthy behaviors in adolescents at follow-up.

\section{Self-administered SSIs since 2016}

Depression. Four randomized SSI trials have examined effects on depressive symptoms since January 2016. One computerized, parent-directed SSI used psychoeducation techniques to communicate evidence-based parenting practices and provide personalized parenting feedback to prevent adolescent internalizing symptoms. Authors observed no significant reductions in adolescent depressive symptoms at a 3-month follow-up, versus a waitlist control ( $d s=0.12$ per youth report, -0.04 per parent report) (Cardamone-Breen et al., 2018). A separate study evaluated whether a computerized, 30-40 minute SSI using motivational interviewing techniques could reduce depressive symptoms at 3, 6, and 12-month follow-ups among adolescents presenting to an emergency department (Ranney et al., 2017). SSI-group adolescents reported lower depressive symptoms at 3 and 12 month follow-ups ( $d=0.19$ at 3 months) but not 6-month follow-up $(d=0.08)$, versus youths who received a list of local resources. Finally, webbased youth-directed SSIs teaching growth-mindset (the belief that personal traits are malleable through personal effort) reduced adolescent depressive symptoms at 9-month and 4-month follow-ups, respectively, compared to time-matched, online control programs (Schleider, Burnette, Widman, Hoyt, \& Prinstein, 2019; Schleider \& Weisz, 2018). These trials were conducted in (a) high-symptom adolescents $(d \mathrm{~s}=.32$ per youth report, .60 per parent report $)$, and (b) adolescent girls attending rural, low-SES high schools $(d=.23$ via youth report). 
Anxiety. One growth mindset SSI trial observed reductions in parent-reported youth anxiety at 9months, compared to the active control program; however, there were no significant reductions in youthreported youth anxiety at the 9-month follow-up (Schleider \& Weisz, 2018). Additionally, this SSI did not significantly reduce adolescent social anxiety in the growth mindset SSI group versus the active control group $(d=0.21$; Schleider et al., 2019). One parent-directed SSI using psychoeducation about parenting skills found no significant reductions in adolescent depressive symptoms 3-months later, versus a waitlist control ( $d s=0.18,-0.06$ per youth and parent reports; Cardamone-Breen et al., 2018).

Externalizing. Only one SSI trial evaluated intervention effects on externalizing symptoms. In this study, adolescents reported no reductions in conduct problems at a 4-month follow-up compared to a control group, who received an active, time-matched comparison SSI ( $d=.01$; Schleider et al., 2019).

Other. In two trials of growth mindset SSIs, significant effects on proposed intervention mechanisms were observed. In one trial, adolescents receiving a growth mindset SSI (versus a control SSI) reported greater increases in growth mindset, primary perceived control, and perceived secondary perceived control immediately post-intervention (Schleider \& Weisz, 2016). Effects on primary perceived control, but not secondary control, persisted at a 9-month follow-up (Schleider \& Weisz, 2018). In the second trial, adolescents reported stronger growth mindset (as opposed to fixed mindset) after receiving a growth mindset SSI relative to their active control-group peers (Schleider, Burnette, et al., 2019).

Summary. The youth SSI literature has grown notably since Schleider and Weisz's (2017) metaanalysis. First, 7 of 8 new SSI trials targeted adolescents, who previously showed smaller symptom reductions following SSIs than younger children (meta-analytic $g \mathrm{~s}=0.42$ and 0.19 for children and adolescents, respectively) — and 5 of 8 new SSI trials targeted depression, relative to 6 of 50 prior trials. Thus, new results reflect considerable growth in the study of adolescent- and depression-focused SSIs. Indeed, these trials suggest the promise of at least two web-based SSIs, and at least one therapistdelivered SSI, for reducing depressive symptoms in adolescents 4- to 12-months later (Ranney et al., 2017; Schleider \& Weisz, 2018; Schleider et al., 2019). It is also notable that no new trials have included SSIs explicitly targeting externalizing youth problems, although one new trial suggested that a growth 
mindset SSI failed to reduce adolescent conduct problems (Schleider et al., 2019). This result aligns with those of the 2017 meta-analysis: SSIs targeting youth conduct problems have shown positive effects overall $(g=.54)$, but most of the effective SSIs targeted parents rather than youths and emphasized elements of behavior parent training rather than individual cognitions (Schleider \& Weisz, 2017). Likewise, the new SSI trials showed mixed benefits for anxiety in youth, despite the significant and positive meta-analytic for anxiety-targeted SSIs observed previously $(g=.56)$. This may reflect the fact that effective anxiety-focused SSIs have largely emphasized graded exposure, widely viewed as an “active ingredient" in evidence-based anxiety treatment (Chorpita \& Daleiden, 2009).

Combined, the results of Schleider and Weisz's meta-analysis and results of trials conducted since suggest the promise of certain SSIs for reducing youth internalizing and externalizing problems. They also suggest useful avenues for formalizing a rigorous approach to SSI evaluation, and ultimately, optimizing their potential to effect positive change. Below, we outline recommendations for next-steps in the study of SSIs, with a focus on three related areas: Future directions in developing SSIs, including a summary of the approach our team has applied to design three different web-based SSIs; future directions in evaluating SSIs; and future directions in adapting and implementing SSIs to meet the needs of presently underserved populations, and to sustainably diffuse effective SSIs into the mental healthcare ecosystem. Future Directions in Developing SSIs

SSIs broadly involve the intentional delivery of a single-encounter program, but numerous approaches exist for specifying the context of SSIs. Several models for conducting face-to-face, therapistdelivered SSIs are detailed by Hoyt and colleagues (2018), including solution-focused, behavioral, and narrative approaches to SSI design and delivery. Our team's primary focus is on web-based SSIs, which are designed for self-administration by youths in a single sitting. Not only have web-based SSIs demonstrated levels of efficacy similar to face-to-face SSIs (Schleider \& Weisz, 2017), but they also have high potential for scalability, as they can be made openly available online for youths to complete in any location. Below, we describe our approach to designing web-based SSIs. 
The B.E.S.T. elements of SSIs for youth mental health. We use four primary elements to guide the development of SSIs for youth mental health. These components were drawn from basic research from social psychology (Lewin, 1944; Aronson, 1990) and qualities common to self-administered SSIs and brief interventions that have shown promising effects (e.g., Schleider \& Weisz, 2018; Miu \& Yeager, 2015; Schleider et al., 2019; Schleider, Mullarkey, \& Chacko, 2019):

(1) B: Brain science to normalize concepts in the program

(2) E: Empower youths to a "helper" or "expert" role

(3) S: Saying-is-believing exercises to solidify learning

(4) T: Testimonials and evidence from valued others

Brain science to normalize concepts. To enhance message credibility, and to encourage youths to perceive their experiences as normative, each SSI we have designed incorporates ideas from brain science to explain key portions of program content. For instance, our SSI teaching self-compassion, "Teen Goals Project," teaches participants that the "pattern-matching part of the brain"—while usually helpful—can occasionally detect patterns that are not true (e.g., associating "more self-criticism" with "higher odds of success"). Likewise, our SSI teaching the belief that personal traits are malleable, called "Project Personality," recruits the concept of neuroplasticity to explain why and how our emotions, symptoms, and coping skills can change (consistent with other, similar interventions; e.g., Miu \& Yeager, 2015; Schleider et al., 2019). We emphasize, in both cases, that all human brains operate in these ways: that is, all of us have some capacity for growth, all of our brains occasionally detect patterns that are not true. In addition to piquing youths' interest, we believe that incorporating brain science-rooted explanations (1) renders the SSIs' messages easier for participants to trust as universally true, despite the personal challenges they might have experienced; and (2) helps normalize the SSIs' messages.

Importantly, some literature suggests that certain types of biological explanations of mental illness — such as "depression is a chemical imbalance" or "mental illness is genetically determined"may have adverse effects, such as increasing stigma against individuals with psychiatric disorders (Loughman \& Haslam, 2018). The "brain science" explanations in our SSIs differ in important ways 
from those that have been identified as potentially iatrogenic. First, we focus on the brain's potential to grow and change by altering one's own actions and thoughts. Indeed, virtually all biological functions are characterized by both stability and malleability; biological systems are responsive to the environment and, in the case of the brain, are highly plastic over time (Turrigiano \& Nelson, 2004). Thus, we use brain science to promote a prospective, internally controllable, and unstable view of one's own neurobiologya specific frame shown to promote positive rather than adverse social-emotional responses (MacDuffie \& Strauman, 2017). SSI participants are oriented not to whether they could have prevented the onset of their difficulties, but rather to their capacity to personally alter the future course of those difficulties.

Empower youths to an expert role. Article 12 of the UNICEF Convention on the Rights of the Child states that when adults are making decisions that affect children, children have the right to express opinions and have those opinions taken into account (U.N. General Assembly, 1989). Participatory action researchers have long proposed that, to optimize acceptability of youth-directed interventions, it is necessary to recruit and respond to youths' expertise and ideas (Baum, MacDougall, \& Smith, 2006). Beyond strengthening youths' investment in new treatments, highlighting youths' active involvement and contributions within a program may strengthen youths' feelings of competence, agency, and relatednessall of which are core to positive social-emotional outcomes (Berg, Coman, \& Schensul, 2009; Samdal \& Rowling, 2015). As such, in all of our SSIs, youths are treated as experts rather than passive recipients of treatment. As one example, the initial portion of "The A.B.C. Project," our SSI teaching behavioral activation (Schleider, Mullarkey, Mumper, \& Sung, 2019), begins as follows:

"We need your help! We are scientists from Stony Brook University. We study the brain, emotions, and how teens cope with setbacks and stress. Every day, we work with kids and teens going through challenges in their lives, like dealing with worry, sadness, and stress. All teens experience these things at some point. So we are always trying to find new ways to help. Other teens have told us that they have found this activity interesting and helpful. But we need your help explaining it in a better way to help 
more kids like you. Please help us and other teens like you by completing this activity carefully."

Youths' written responses and advice to peers in The A.B.C. Project will be used to inform and develop and improve our SSIs. Youth participants are, in a sense, co-constructors of the SSIs we design. We believe this frame helps bolster youths' sense of agency and ownership during SSI completion.

Saying-is-believing activities to solidify learning. "Saying-is-believing" activities are designed to promote internalization of novel beliefs or ideas via self-persuasive writing exercises. They are drawn from social psychological theory (Aronson, 1999) and have been included in numerous educational interventions for adolescents (e.g., targeting achievement motivation; Aronson, 1999; Yeager \& Walton, 2011). The activity consists of two parts. First, the participant is asked to write in detail about a setback, stressor, or struggle relevant to the SSI's main message, per within-program prompts. Next, the participant is asked to offer advice to a peer who is now facing that same stressor, explaining to the peer how she might use the SSI's message to cope with the stressor at hand. For example, in the behavioral activation SSI noted above (Schleider et al., 2019), the first Saying-is-Believing prompt asks the participant to write about a time when a "roadblock thought" - a negative, inaccurate thought about one's ability to take positive action, which the participant identifies earlier in the program — prevented them from taking action towards an important goal. Next, the participant is asked to advise a peer on how they might act opposite to that same roadblock thought, using newly-gleaned knowledge from the SSI. As research on "saying-is-believing" activities suggests (Aronson, 1999), advocating for a persuasive message to a personally-relevant audience is a powerful means of increasing one's own belief in that message.

Testimonials and evidence from valued others. The utility of testimonials (personal narratives) has received considerable attention among health communication researchers (Hamby, Daniloski, \& Brinberg, 2015). Personal testimonials are thought to capture audiences' attention by engaging readers both cognitively and emotionally. Meta-analyses suggest that testimonials increase the persuasiveness of health-related messaging (Shen et al., 2015; van Laer, Feiereisen, \& Visconti, 2019), including messaging 
around mental health (Johnson, Quintero Johnson, Yilmaz, \& Najarian, 2017). For youths, the persuasive power of testimonials appears more pronounced when narratives come from peers, near-peer role models, or 'experts' in a given domain (Cody, 2017; van Laer et al., 2019; Borah \& Xiao, 2018). As such, our SSIs include testimonials — stories from older peers and 'experts' (typically scientists) — to illustrate the program's core ideas. As an example, Figure 1 includes portions of "The A.B.C. Project" (Schleider et al., 2019) in which "Kat" shares her story about acting opposite to depression.

Notably, the B.E.S.T. elements reflect those that our team has used to guide SSI development. They are present in many brief interventions that have reduced psychopathology in youth (Schleider, Mullarkey, \& Chacko, 2019), but they are not present in all SSIs that have shown promise. Thus, we offer this framework as a potentially helpful template for others interested in developing novel SSIs, but the extent to which they are collectively or individually necessary is unknown. Such questions will be valuable to investigate in future research-for instance, in trials comparing SSIs that do and do not include specific B.E.S.T. elements.

\section{Future Directions in Evaluating SSIs}

Realizing SSIs' potential to support youth mental health at scale will require rigorous, consistent standards for evaluating their effects. Accordingly, we propose five recommendations for future evaluations of SSIs for youth within randomized trials, which we believe apply across SSI delivery formats. These recommendations are based on three ideas: 1) valid and reliable measurement and preregistration of methods are essential to properly evaluate SSIs; 2) any strong claims about SSIs require commensurately strong, relevant control conditions; and 3) changes from immediately pre- to immediately-post SSI have important utility.

Recommendation 1. Choose measures carefully. Claims about SSIs' effects rest on the reliability and validity of chosen assessments. Questionable measurement practices, or decisions researchers make that leave unanswered questions about the measurement approach, can undermine reliability and validity (Flake \& Fried, 2019). For example, developing scales "on-the-fly" —which may be tempting when evaluating novel interventions - can lead to high apparent psychometric acceptability 
(i.e., high internal consistency) without corresponding evidence for construct validity (Flake, Pek, \& Hehman, 2017), rendering hard-to-interpret results. Therefore, we encourage the use of validated, brief assessments, ideally assessing targets a given SSI is predicted to shift. Clinical psychologists may be especially well-positioned to forward this goal in future SSI trials. Many existing SSIs have been designed and evaluated by scientists in neighboring fields to clinical science (e.g., social psychology; education); such studies have attended to mental health-relevant effects, but with varying reliance on well-validated assessments of clinical outcomes. To optimize precise understandings of mental health SSI' effects, we strongly recommend that future trials rely on well-validated symptomatology assessments (for open-source, brief, well-validated measurement options, see Beidas et al., 2015). Strengthening quality of mental health assessments may have multiple effects - from generating more prescise estimates of SSIs' effects to increasing statistical power to detect interventions' impacts when they exist (Markon, Chmielewski, \& Miller, 2011).

Recommendation 2. Specify methods in advance via pre-registration. Pre-registering a study involves detailing your hypotheses, specific research design, and analytic plan prior to beginning a clinical trial. Prospective pre-registration of clinical trials has long been required by the International Committee of Medical Journal Editors, the National Institutes of Health, and other agencies; however, existing pre-registration templates (e.g., clinicaltrials.gov) do not presently require scientists to outline all features of one's analytic plan prior to starting a study (e.g., detailing how analytic 'choice-points' will be addressed). We strongly recommend that prospective pre-registrations of SSI trials include a detailed analytic plan, including how scores will be calculated and how intervention effects will be tested (Benning, Bachrach, Smith, Freeman, \& Wright, 2018). Doing so helps to lower risk of false-positive results and other questionable research practices such as outcome-switching, or changing which measure was the "primary target" of the intervention following data analysis (Boutron, Dutton, Ravoud, \& Altman, 2010).

Recommendation 3. Use plausible control conditions. Using strong, relevant control conditions will vastly improve certainty about the specificity and strength of an SSI's effects. With regard to 
strength, we suggest that control conditions be presented as credible treatments rather than alternative, unrelated assignments or wait-lists (American Psychological Association, 1995). In traditional clinical trials, wait-list conditions are often thought to rule out whether effects might be attributable to the passage of time. Since so little time passes during SSI administration, such comparison conditions may have limited utility. Some previous SSI trials have used explicitly non-therapeutic, educational materials as controls (e.g., Miu \& Yeager, 2015). While such materials are preferable to wait-lists, they yield less certainty that observed effects are specific to the particular mental health SSI being delivered. Comparisons to credible placebo controls - that is, those designed to resemble therapeutic activities, and that include generally positive messaging, but that are not designed to shift the same target as the SSI at hand - will allow us to determine whether an SSI is more therapeutic than a condition that might evoke similar expectations of improvement. With regard to relevance, control conditions should match the SSI as closely as possible on features that are not hypothesized to be active, therapeutic elements. For example, an SSI containing a self-persuasion writing exercise (Aronson, 1999) might have a matched control with a more generic writing exercise. Matching these design features closely will allow conclusions about the effects of the SSI to be even more specific to the therapeutic elements included. Future research might also dismantle SSIs into their components and directly test which individual SSI elements, and their combinations, are most effective. To support future researchers interested in evaluating web-based SSIs, all materials for an "active control" SSI we have used in multiple studies are available for others to use in their work (https://osf.io/swv5b/; Schleider \& Weisz, 2019).

\section{Recommendation 4. Optimize SSIs' precision and predictive power by assessing immediate}

outcomes. We strongly suggest evaluating immediate post-SSI outcomes, not just longer-term symptom outcomes, in future SSI trials. Immediate post-SSI assessments of proximal outcomes can both enable manipulation checks and mechanism-testing. Manipulation checks allow us to determine whether the SSI successfully targeted a proximal, theoretically-informed outcome of interest (e.g., hopelessness). These manipulation checks can help gauge whether the SSI's intended message was internalized by participants, and may be especially important during the early stages of SSI development. Likewise, in larger-scale and 
longer-term tests of SSIs, variables used in manipulation checks may remain important for at least two reasons. First, improvements in later outcomes (i.e., symptoms) despite a failed manipulation check could indicate the SSI is not operating through predicted pathways. Second, variables assessed via manipulation checks may serve as key predictors or mechanisms of SSIs' future effects. Thus, we encourage the assessment of multiple theoretically-informed constructs immediately post-SSI—focusing on proximal outcomes that may account for the intervention's longer-term utility - that could present plausible mechanisms for the SSIs' future effects on symptoms. For example, greater changes immediately post-SSI in secondary perceived control predicted greater reductions in anxiety over the course of nine months, whereas greater changes in growth mindset of personality did not (Schleider, Abel, \& Weisz, 2019). Assessing these potential mechanisms at pre- and post-SSI also allows for responsible mediation testing, with changes in a mediator preceding the changes in distal outcomes (Kazdin, 2007). It also may facilitate work differentiating proximal and distal SSI outcomes - that is, whether SSIs tend to shape certain outcomes (e.g., hope; agency) in the immediate term, but other outcomes (e.g., depression symptoms; overall well-being) in the longer-term. Indeed, some evidence supports 'sleeper effects' following SSIs, where symptom changes emerge months after an SSI but changes in other outcomes are detectable immediately (Schleider \& Weisz, 2016; Schleider \& Weisz, 2018). Some have suggested that brief interventions may exert 'delayed' impacts on outcomes of interest by triggering recursive learning processes. For instance, an SSI might ignite initial belief change, but subsequent behavior change emerges following real-world experience and practice applying those new beliefs (Yeager \& Walton, 2011). This possibility is ripe for investigation in future studies.

These responsible mediator tests are greatly needed, as the factors underlying specific SSIs' effects remain poorly understood. Fortunately, web-based SSIs' relatively high potential for accessibility and scalability allows us to conduct studies that are properly-powered to compare contributors to SSI effectiveness - for instance, by conducting SSI trials entirely online (Schleider \& Weisz, 2018). Indeed, web-based SSIs are well-positioned for testing as massive open online interventions (Muñoz et al., 2016), which enable rapid recruitment of hundreds or thousands of participants. Such studies may allow for 
direct tests of whether longer-term symptom improvements result from immediate increases in perceived control, decreased hopelessness, or increased change expectancies immediately following an SSI. The potential for testing mediators at scale is an advantage over traditional psychotherapy trials, which are typically under-powered to differentiate impacts of common or specific contributors to change (Cuijpers, Reijnders, \& Huibers, 2019). Some initial potential mediators with empirical support include primary perceived control and secondary perceived control (Schleider, Abel, et al., 2019). Other proximal outcomes that may be relevant in SSIs include hopelessness and agency (Dryden, 2018)—possibilities that we are evaluating in ongoing studies (Schleider, Mullarkey, \& Weisz, 2019; Schleider, Dobias, \& Pati, 2019). Relevant targets will likely vary across SSIs, and pilot testing using mixed-methods approaches could help identify youths' perceptions of whether and why they found a specific SSI helpful. Such work will allow us to better understand participants' perceptions as "common" across different SSI experiences. Factors experienced as "common" by SSI participants may differ from the factors experienced as "common" during traditional, longer-term psychotherapy (e.g., therapeutic alliance). Ultimately, this type of mediator-testing may set the stage for better-informed matching of youths to SSIs most likely to benefit them, both in the immediate and longer-term.

\section{Recommendation 5. Capitalize on within- and between-person data to improve prediction}

of SSI response. Prediction-focused approaches that explicitly seek to identify "best-responders" to a particular SSI could also facilitate effective treatment-matching (Cohen \& DeRubeis, 2018; Dwyer, Falkai, \& Koutsouleris, 2018). A challenge, of course, involves identifying which pre-treatment factors are most useful as predictors of SSI response. These approaches also focus on generalizing their predictions to new samples rather than maximizing fit in the current sample (Yarkoni \& Westfall, 2017). Given that a major strength of SSIs is their scalability, this prioritization of out-of-sample performance could be especially useful. The scalability and accessibility of many SSIs can also allow for data that is observation and feature-rich2. In other words, we can collect many potentially relevant predictor variables

2 Also, contrary to some views, variable selection, or feature engineering, is a key element of creating these predictive models. We encourage investigators to assess variables they believe will predict 
from many youth, which increase the utility of powerful prediction-focused approaches such as neural networks (Makridakis, Spiliotis, \& Assimakopoulos, 2018). Another approach to creating feature- and observation-rich data is to collect many observations $(N>100)$ from a particular youth before the intervention begins, via ecological momentary assessment. These data can be evaluated using idiographic, or individual level, methods (Piccirillo \& Rodebaugh, 2019) and then used to predict response to treatment (Lutz et al., 2018). For instance, youths with certain symptom structures, patterns, or profiles might respond better to specific types of SSIs; collecting symptom-level data at many time-points prior to SSI administration could ascertain this possibility. Ultimately, both between-subject and within-subject feature-rich, observation data could facilitate modeling approaches well equipped to help match youths to SSIs most likely to reduce their presenting problems.

Recommendation 6. Make SSI program materials open-source. Openly sharing interventions and data whenever possible could further improve our ability to predict who will respond best to which SSI. We have shared materials for three of our web-based SSIs; adolescents may complete and provide feedback on as part of an ongoing, anonymous program evaluation project (www.schleiderlab.org/yes), and research can freely access the materials for research purposes (Schleider \& Weisz, 2019, https://osf.io/259jv/; Schleider, Mullarkey, Mumber, \& Sung, 2019, https://osf.io/qj94c/; Dobias, Mullarkey, \& Schleider, 2019, https://osf.io/ydqxj/). We encourage others to make their materials available as well to optimize the rate of discovery with respect to SSIs — most critically, identifying when and for whom they do and do not help. In the long-term, SSI investigators could follow the model of Psychological Science Accelerator (Moshontz et al., 2018) and collaborate on large-scale interventions with harmonized measures across many sites. This model, along with pre-registration of analysis plans to avoid over-fitting, could help identify specific, robust contextual factors that may impact intervention response beyond individual-level characteristics.

intervention response before the SSI and then test those intuitions using these prediction-focused approaches. Even in smaller samples $(N<300)$ prediction-focused techniques such as the elastic net, an out-of-sample prediction optimizing version of the familiar linear regression (Zou \& Hastie, 2005), can provide prediction-focused alternatives to traditional statistical techniques. 


\section{Future Directions in Adapting and Implementing SSIs}

Recommendation 1: Assess Stakeholder views of SSIs. As SSIs are further investigated, future studies should actively explore ways to optimize the translation of SSIs from the lab to clinical and community settings. While SSIs' flexibility and brevity overcome some common access barriers to implementing traditional evidence-based treatments (EBTs; e.g. extensive training requirements or limits on clinicians' time), SSIs cannot circumvent all barriers. Sanders and Turner's (2005) systems-contextual perspective suggests that we must consider all aspects of quality training, practitioner variables, client variables, and organizational support for successful dissemination and implementation of any new evidence-based practice.

Thus, an important preparatory step to successful translation of SSIs may involve assessing various stakeholders' views of SSIs. These stakeholders may include therapists, clients, parents, administration staff, school staff, policy makers, and insurance companies. Stakeholder opinions should be incorporated from the start of any SSI implementation effort to ensure that the program is structured to meet the community's needs, rather than those perceived by the researcher. Stakeholders and scientists might differ, for instance, in preferences about which SSIs might be best-fit to a particular community, where and by whom those SSIs should be delivered, and what outcomes are most important to assess. Indeed, the outcomes of interest may drastically differ as a function of informant (Cuijpers, 2019). Consistent with tenets of community-based participatory research, we believe that integrating stakeholders' perspectives will increase the likelihood of an SSI's success and facilitate reciprocal learning and thus mutual benefit for all involved (Wallerstein \& Duran, 2010).

Additionally, we encourage researchers to pay special attention to any negative beliefs that may impede the uptake of SSIs so that future dissemination and implementation efforts can specifically target or address these beliefs. To date, no study has assessed stakeholders' assumptions, expectations, or beliefs about SSIs' effectiveness. A helpful first step may be understanding families' and therapist's perceptions of SSIs, as they may directly influence acceptability and feasibility of implementation attempts. At the same time, we recommend evaluating how organizational structures may impact clinicians' use of SSIs to 
gauge the appropriate level of support required to sustain consistent use. Future studies may benefit from mixed methods approaches to assess for these variables and, more importantly, explore promising pathways to improving SSI uptake outside of research contexts.

Recommendation 2: Evaluate SSIs in high-acuity populations. There is no evidence to suggest SSIs can exclusively benefit youths with mild-to-moderate, low-acuity symptoms. Adolescents can benefit from brief, targeted interventions. Although across 50 randomized trials of SSIs, intervention effects were not moderated by youth symptom severity (Schleider \& Weisz, 2017), relatively few SSIs have targeted high-severity and acute problems in children and adolescents. Here, we use self-injurious thoughts and behaviors (SITBs) as an exemplar of a higher-severity outcome that may be malleable via brief intervention. Developing brief, scalable interventions for this population is of critical importance: nearly $66 \%$ of youths experiencing suicidal thoughts do not access treatment (Husky et al., 2012), in part due to parents' limited awareness of if, and when, their child experiences suicidal ideation (Jones et al., 2019). SSIs for SITBs may offer new opportunities to improve high-need youths' access to support. Specifically, SSIs may facilitate intervention delivery during critical temporal periods where risk spikes. For example, risk of suicide skyrockets during the period following psychiatric hospitalization, with an estimated suicide rate more than 3 times the rate estimated among inpatients (Chung et al. 2017; Walsh, Sara, Ryan, \& Large, 2015). Recent research has used machine learning and real-time monitoring methods to identify other potential high-risk periods for SITBS (Kleiman, Glenn, \& Liu, 2019; Kleiman \& Nock, 2018). The SSI mode of treatment delivery has great potential for future combination with these risk monitoring techniques; after identifying those at elevated risk, SSIs could deliver intervention content precisely when it is needed.

Indeed, preliminary evidence suggests brief interventions can alleviate adolescent SITBs. A metaanalysis suggested that asking about suicide, or exposure to suicide-related content, during research studies was linked with small, significant reductions in suicidal ideation ( $g=-.13$; Blades, Stritzke, Page, \& Brown, 2018). Reductions in suicidal ideation post-suicide related exposure were twice as large for adolescents $(g=-.22)$ than for adults; thus, simply asking adolescents about suicide serves as a brief 
intervention to alleviate ideation (Blades et al., 2018). Among older adolescents endorsing active suicidal ideation, a 35-minute video outlining problem-solving techniques produced initial reductions in selfreported ideation, versus an active control (Fitzpatrick, Witte, \& Schmidt, 2005). While effects of this SSI were short-lived, it is notable that a single video produced any detectable reductions in ideation.

Brief interventions may impact other suicide-related outcomes beyond suicidal ideation. In a large sample of 448 adolescents with past-month suicide ideation or attempt, teenagers who nominated “caring adults" to act as a future, formal "support team" experienced a lower group mortality rate and were more likely to access outpatient mental health treatments across an 11-to-14 year follow-up period (King et al., 2019). Intervention specialists maintained weekly check-in phone calls with nominated adults for the first 3 months; however, the primary training for these adults involved just one 60-minute session. This study is a prime example of how brief, light-touch interventions may improve suicide outcomes via a wide variety of delivery formats. Assessing SITBs in future SSI trials may help identify characteristics of other brief programs that might exert similar benefits.

\section{Recommendation 3: Test whether SSIs can reduce wait-times for outpatient psychotherapy.}

Even among those with health insurance living in cities with high densities of providers, barriers to accessing outpatient mental health services are great. In one study, just $33 \%$ of investigators' simulated "patient" calls to mental health service providers in Boston, Chicago, and Houston were answered on a first attempt; $64 \%$ of unanswered phone calls were never returned, and just $24 \%$ resulted in a scheduled appointment (Malowney, Keltz, Fischer, \& Boyd, 2015). Likewise, less than one third of Canadian youth mental health agencies reported meeting the Canadian Psychiatric Association wait-time benchmarks (Kowalewski, McLennan, \& McGrath, 2011). Given that longer wait-times for psychotherapy predict worse clinical outcomes once treatment is accessed (i.e., a 'nocebo' effect; Furukawa et al., 2014), there is a need for service delivery models that facilitate rapid access to care. Providing SSIs in outpatient settings, either on a walk-in or by-appointment basis among treatment-seeking clients placed on a waiting-list for longer-term treatment, may help address this need. This approach may have the added 
benefit of reducing waitlist lengths: if some youths find an SSI sufficient in spurring symptom change, they may choose to forego longer-term treatment, decreasing wait-times for others.

Presently, SSI services in outpatient are rare within the United States (Hoyt et al., 2018), but there are some notable examples. The Austin Child Guidance Center in Austin, Texas offers a walk-in singlesession counseling service for youths, with the goal of providing immediate access to care. Separately, our team is testing the efficacy of a single-session consultation program, based on solution-focused brief therapy principles, for adolescents and adults at seeking psychotherapy at the Krasner Psychological Center at Stony Brook University (clinicaltrials.gov, NCT04069832). Participants will receive a one-hour, solution-focused consultation within two weeks of contacting the clinic. We will test the program's effects on clients' hope and agency, along with whether the clinic's waitlist is reduced from before to after providing the consultation service. Examining the potential of SSIs to reduce waitlist burden represents a promising area for future study.

\section{Recommendation 4: Examine Diverse SSI Implementation Opportunities}

Per our above-mentioned definition of SSIs, their brief, mechanism-targeted nature allows for their creative application across many existing service settings. This flexibility may be useful in settings with limited resources to provide individual, face-to-face, multi-session psychotherapy. In fact, SSIs have been frequently evaluated in nontraditional settings for mental health treatment delivery, including primary care, emergency rooms, schools, and community centers (Schleider \& Weisz, 2017), rendering them accessible to many more youths than those who access specialty mental health care. Likewise, selfadministered, web-based SSIs allow individuals to access therapeutic tools anywhere at any time, further increasing their adaptability (Cardamone-Breen et al., 2018; Ranney et al., 2017; Schleider \& Weisz, 2018). Future possibilities for optimizing this potential include personalizing web-based SSIs as "just-intime" interventions or matching youths to targeted SSIs based on symptom profiles.

An additional future direction might involve training lay mental healthcare providers (e.g., schoolteachers, pediatricians, mentors) to facilitate youth-focused SSIs. Because they are on the "frontlines" in caring for vulnerable youth, lay providers are ideally-positioned to identify youths who 
might benefit from an SSI, and to deliver that SSI at opportune moments (Brown, Green, Desai, Weitzman, \& Rosenthal, 2014; Rothì, Leavey, \& Best, 2008) . This notion of task-sharing, or the practice of delegating specific mental health service delivery tasks to lay providers, is not new; this model has been tested as an acceptable means of helping to reduce treatment gap . Common barriers to task-sharing efforts include lay providers' feeling ill-equipped to deliver mental health interventions (Hung et al., 2014; Mendenhall et al., 2014) ongoing support and supervision (Javadi, Feldhaus, Mancuso, \& Ghaffar, 2017). Fortunately, SSIs' structure alleviates several of these concerns: many SSIs require little to no training to administer, and several can be completed independently by youths in less than one hour. As such, offering lay providers access to a suite of SSIs, which they may flexibly offer to offer clients, mentees, or students, could be highly synergistic with their existing goals and tasks. Given the sheer number of lay providers relative to trained therapists, and the potential impact of equipping them with SSIs on service access and utilization, these possibilities merit formal investigation.

\section{Recommendation 5: Conduct cost-effectiveness evaluations within SSI trials.}

In the United States, psychiatric disorders rank as the costliest health-related conditions to treat (at least \$201 billion annually; Roehrig, 2016). Because resources for treating youth psychopathology are limited and are growing more constrained with increased service demand (Mojtabai, Olfson, \& Han, 2016), cost-effectiveness analyses (CEAs) and cost-utility analyses (CUAs) of various treatments are increasingly critical. In the case of SSIs, CEAs and CUAs may be especially informative: Although it is likely that SSIs confer fewer costs than traditional psychotherapies, the magnitude of this difference is unknown, as is cost-to-gain ratio for SSIs versus longer-term therapies targeting similar problems. CUAs or CEAs in SSI trials may help focus implementation efforts on SSIs with strong cost-to-gain ratios.

Both CUAs and CEAs are rare within psychotherapy research (Cuijpers, 2019). In adults, guided internet-based interventions for depression, anxiety, and drug use show greater cost-effectiveness than wait-lists, treatment as usual, and face-to-face cognitive behavioral therapy; likewise, unguided internet treatments for suicide prevention, depression and smoking cessation show greater cost-effectiveness than treatment as usual (Donker et al., 2015). Given promising results for internet-based treatments that vary 
widely in length, it seems worth investigating the relative cost-effectiveness of web-based SSIs versus longer and face-to-face alternatives. Likewise, it may be useful to evaluate cost-effectiveness of integrating an SSI with other treatments. For instance, completing a self-administered SSI before starting weekly psychotherapy may catalyze an adolescent's hope, agency, or positive treatment expectancies-in turn strengthening engagement and clinical response to subsequent treatment. Our team is examining such possibilities in ongoing clinical trials (clinicaltrials.gov, NCT04069832; Schleider, Dobias, \& Pati, 2019), but additional studies using diverse SSIs are needed to gauge this possibility.

\section{Conclusion}

The goal of this article is to introduce, and outline an actionable path towards realizing, the potential of single-session interventions (SSIs) as a force for youth mental health. We defined the scope and nature of SSIs for youth psychopathology; provided an updated review of the literature on SSIs that have and have not reduced mental health difficulties in youth; and proposed recommendations for designing, evaluating and learning how to implement SSIs in a variety of settings and contexts. It is critical to note that we do not view SSIs as a "silver bullet" - either for meaningfully reducing levels of youth psychopathology or for closing the mental health treatment gap. As has been noted previously (Chorpita, 2019), forwarding these objectives will require diversification of accessible treatment options, including services of varying lengths, formats, and intensities. Indeed, many youths will certainly require or benefit from longer-term services. Nonetheless, SSIs offer a framework for scaling-up many types of potentially helpful supports, as they can be designed to target a wide variety of skills, ways of thinking, and problem types. Pursuing such work may improve youths' odds of accessing some evidence-based support when the modal alternative is no support at all. Additionally, web-based and self-administered SSIs allow for rapid dissemination, evaluation, and iterative improvement, based on data from much larger samples than are typically feasible via clinical trials of lengthier and face-to-face treatments. As work on SSIs progresses, identification of individual-level predictors of treatment response - and the prospect of matching youths with SSIs likely to benefit them most-may become real possibilities. We 
hope these recommendations will spur a new wave of SSI research among clinical intervention scientists, and that cumulative findings help reduce the need-to-access gap for youths worldwide. 


\section{References}

American Psychological Association. (1995). Task Force on Psychological Intervention Guidelines. Template for Developing Guidelines: Interventions for Mental Disorders and Psychosocial Aspects of Physical Disorders.

Aronson, E. (1999). The power of self-persuasion. The American Psychologist, 54(11), 875.

Baum, F., MacDougall, C., \& Smith, D. (2006). Participatory action research. Journal of Epidemiology and Community Health, 60(10), 854-857.

Beidas, R. S., Stewart, R. E., Walsh, L., Lucas, S., Downey, M. M., Jackson, K., ... \& Mandell, D. S. (2015). Free, brief, and validated: Standardized instruments for low-resource mental health settings. Cognitive and Behavioral Practice, 22, 5-19.

Benning, S. D., Bachrach, R. L., Smith, E., Freeman, A., \& Wright, A. G. C. (2018). The Registration Continuum in Clinical Science: A Guide toward Transparent Practices. PsyArXiv. https://doi.org/10.31234/osf.io/an8h3

Berg, M., Coman, E., \& Schensul, J. J. (2009). Youth Action Research for Prevention: a multi-level intervention designed to increase efficacy and empowerment among urban youth. American Journal of Community Psychology, 43(3-4), 345-359.

Blades, C. A., Stritzke, W. G. K., Page, A. C., \& Brown, J. D. (2018). The benefits and risks of asking research participants about suicide: A meta-analysis of the impact of exposure to suicide-related content. Clinical Psychology Review, Vol. 64, pp. 1-12. https://doi.org/10.1016/j.cpr.2018.07.001

Borah, P., \& Xiao, X. (2018). The Importance of "Likes": The Interplay of Message Framing, Source, and Social Endorsement on Credibility Perceptions of Health Information on Facebook. Journal of Health Communication, 23(4), 399-411.

Boutron, I., Dutton, S., Ravaud, P., \& Altman, D. G. (2010). Reporting and interpretation of randomized controlled trials with statistically nonsignificant results for primary outcomes. JAMA, 303, 2058-64.

Brown, N. M., Green, J. C., Desai, M. M., Weitzman, C. C., \& Rosenthal, M. S. (2014). Need and unmet 
need for care coordination among children with mental health conditions. Pediatrics, 133, e530-537.

Calvete, E., Fernández-Gonzalez, L., Orue, I., Echezarraga, A., Royuela-Colomer, E., Cortazar, N., ... Yeager, D. S. (2019). The Effect of an Intervention Teaching Adolescents that People can Change on Depressive Symptoms, Cognitive Schemas, and Hypothalamic-Pituitary-Adrenal Axis Hormones. Journal of Abnormal Child Psychology, 47, 1533-

1546.file:///Users/jessica.schleider/Downloads/, Vol. 47, pp. 1533-1546. https:/doi.org

Cardamone-Breen, M. C., Jorm, A. F., Lawrence, K. A., Rapee, R. M., Mackinnon, A., \& Yap, M. (2018). A Single-Session, Web-Based Parenting Intervention to Prevent Adolescent Depression and Anxiety Disorders: Randomized Controlled Trial. Journal of Medical Internet Research, 20, e148.

Cartwright-Hatton, S., Ewing, D., Dash, S., Hughes, Z., Thompson, E. J., Hazell, C. M., ... Startup, H. (2018). Preventing family transmission of anxiety: Feasibility RCT of a brief intervention for parents. British Journal of Clinical Psychology, 57(3), 351-366.

Chorpita, B. F. (2019). Commentary: Metaknowledge is power: envisioning models to address unmet mental health needs: reflections on Kazdin (2019). Journal of Child Psychology and Psychiatry, $60(4), 473-476$.

Chorpita, B. F., \& Daleiden, E. L. (2009). Mapping evidence-based treatments for children and adolescents: application of the distillation and matching model to 615 treatments from 322 randomized trials. Journal of Consulting and Clinical Psychology, 77(3), 566-579.

Cody, C. (2017). "We have personal experience to share, it makes it real": Young people's views on their role in sexual violence prevention efforts. Children and Youth Services Review, 79, 221-227.

Cohen, J. (2013). Statistical power analysis for the behavioral sciences. Routledge.

Cohen, Z. D., \& DeRubeis, R. J. (2018). Treatment Selection in Depression. Annual Review of Clinical Psychology, 14, 209-236.

Cuijpers, P. (2019). Targets and outcomes of psychotherapies for mental disorders: an overview. World Psychiatry, Vol. 18, pp. 276-285. https://doi.org/10.1002/wps.20661

Cuijpers, P., Reijnders, M., \& Huibers, M. J. H. (2019). The Role of Common Factors in Psychotherapy 
Outcomes. Annual Review of Clinical Psychology, 15(1), null.

de Girolamo, G., Dagani, J., Purcell, R., Cocchi, A., \& McGorry, P. D. (2012). Age of onset of mental disorders and use of mental health services: needs, opportunities and obstacles. Epidemiology and Psychiatric Sciences, 21(1), 47-57.

Dobias, M. L., Mullarkey, M. C., \& Schleider, J. L.(2019). The Teenage Goals Project.Open Science Framework. doi.org/10.17605/OSF.IO/YDQXJ

Dryden, W. (2018). "Single Session Integrated Cognitive Behaviour Therapy" (SSI-CBT). Single-Session Therapy (SST), pp. 289-291. https://doi.org/10.4324/9780429489648-98

Dwyer, D. B., Falkai, P., \& Koutsouleris, N. (2018). Machine Learning Approaches for Clinical Psychology and Psychiatry. Annual Review of Clinical Psychology, 14, 91-118.

Fitzpatrick, K. K., Witte, T. K., \& Schmidt, N. B. (2005). Randomized controlled trial of a brief problemorientation intervention for suicidal ideation. Behavior Therapy, 36, 323-333.

Flake, J. K., \& Fried, E. I. (2019). Measurement Schmeasurement: Questionable Measurement Practices and How to Avoid Them. https://doi.org/10.31234/osf.io/hs7wm

Flake, J. K., Pek, J., \& Hehman, E. (2017). Construct validation in social and personality research: Current practice and recommendations. Social Psychological and Personality Science.

Fox, K. R., Huang, X., Linthicum, K. P., Wang, S. B., Franklin, J. C., \& Ribeiro, J. D. (2019). Model complexity improves the prediction of nonsuicidal self-injury. Journal of Consulting and Clinical Psychology, 87(8), 684-692.

Glenn, C. R., Lanzillo, E. C., Esposito, E. C., Santee, A. C., Nock, M. K., \& Auerbach, R. P. (2017). Examining the Course of Suicidal and Nonsuicidal Self-Injurious Thoughts and Behaviors in Outpatient and Inpatient Adolescents. Journal of Abnormal Child Psychology, 45(5), 971-983.

Hinyard, L. J., \& Kreuter, M. W. (2007). Using narrative communication as a tool for health behavior change: a conceptual, theoretical, and empirical overview. Health Education \& Behavior: The Official Publication of the Society for Public Health Education, 34(5), 777-792.

Hoyt, M. F., Bobele, M., Slive, A., Young, J., \& Talmon, M. (2018a). Single-session/one-at-a-time walk- 
in therapy. Single-Session Therapy by Walk-In or Appointment, 3-24.

Hoyt, M. F., Bobele, M., Slive, A., Young, J., \& Talmon, M. (2018b). Single-Session Therapy by Walk-In or Appointment: Administrative, Clinical, and Supervisory Aspects of One-at-a-Time Services. Routledge.

Hung, K. J., Tomlinson, M., le Roux, I. M., Dewing, S., Chopra, M., \& Tsai, A. C. (2014). Communitybased prenatal screening for postpartum depression in a South African township. International Journal of Gynecology \& Obstetrics, Vol. 126, pp. 74-77. https://doi.org/10.1016/j.ijgo.2014.01.011

Husky, M. M., Olfson, M., He, J.-P., Nock, M. K., Swanson, S. A., \& Merikangas, K. R. (2012). Twelvemonth suicidal symptoms and use of services among adolescents: results from the National Comorbidity Survey. Psychiatric Services , 63(10), 989-996.

Issner, J. H., Mucka, L. E., \& Barnett, D. (2017). Increasing Positive Health Behaviors in Adolescents with Nutritional Goals and Exercise. Journal of Child and Family Studies, 26(2), 548-558.

Javadi, D., Feldhaus, I., Mancuso, A., \& Ghaffar, A. (2017). Applying systems thinking to task shifting for mental health using lay providers: a review of the evidence. Global Mental Health (Cambridge, England), 4, e14.

Johnson, J. M. Q., Quintero Johnson, J. M., Yilmaz, G., \& Najarian, K. (2017). Optimizing the Presentation of Mental Health Information in Social Media: The Effects of Health Testimonials and Platform on Source Perceptions, Message Processing, and Health Outcomes. Health Communication, Vol. 32, pp. 1121-1132. https://doi.org/10.1080/10410236.2016.1214218

Jones, J. D., Boyd, R. C., Calkins, M. E., Ahmed, A., Moore, T. M., Barzilay, R., ... Gur, R. E. (2019). Parent-Adolescent Agreement About Adolescents' Suicidal Thoughts. Pediatrics, Vol. 143, p. e20181771. https://doi.org/10.1542/peds.2018-1771

Kataoka, S. H., Zhang, L., \& Wells, K. B. (2002). Unmet need for mental health care among U.S. children: variation by ethnicity and insurance status. The American Journal of Psychiatry, 159(9), $1548-1555$.

Kazdin, A. E. (2007). Mediators and mechanisms of change in psychotherapy research. Annual Review of 
Clinical Psychology, 3, 1-27.

Kazdin, A. E. (2019). Annual Research Review: Expanding mental health services through novel models of intervention delivery. Journal of Child Psychology and Psychiatry, and Allied Disciplines, 60(4), $455-472$.

Kazdin, A. E., \& Blase, S. L. (2011). Interventions and Models of Their Delivery to Reduce the Burden of Mental Illness: Reply to Commentaries. Perspectives on Psychological Science: A Journal of the Association for Psychological Science, 6(5), 507-510.

Kazdin, A. E., \& Rabbitt, S. M. (2013). Novel Models for Delivering Mental Health Services and Reducing the Burdens of Mental Illness. Clinical Psychological Science, Vol. 1, pp. 170-191. https://doi.org/10.1177/2167702612463566

Kessler, R. C., Demler, O., Frank, R. G., Olfson, M., Pincus, H. A., Walters, E. E., ... Zaslavsky, A. M. (2005). Prevalence and treatment of mental disorders, 1990 to 2003. The New England Journal of Medicine, 352(24), 2515-2523.

Kessler, R. C., \& Wang, P. S. (2008). The descriptive epidemiology of commonly occurring mental disorders in the United States. Annual Review of Public Health, 29, 115-129.

King, C. A., Arango, A., Kramer, A., Busby, D., Czyz, E., Foster, C. E., ... YST Study Team. (2019). Association of the Youth-Nominated Support Team Intervention for Suicidal Adolescents With 11to 14-Year Mortality Outcomes: Secondary Analysis of a Randomized Clinical Trial. JAMA Psychiatry, 76(5), 492-498.

Kleiman, E. M., Glenn, C. R., \& Liu, R. T. (2019). Real-time monitoring of suicide risk among adolescents: potential barriers, possible solutions, and future directions. Journal of Clinical Child \& Adolescent Psychology.

Kleiman, E. M., \& Nock, M. K. (2018). Real-time assessment of suicidal thoughts and behaviors. Current Opinion in Psychology, 22, 33-37.

Konrad, T. R., Ellis, A. R., Thomas, K. C., Holzer, C. E., \& Morrissey, J. P. (2009). County-level estimates of need for mental health professionals in the United States. Psychiatric Services , 60, 
$1307-1314$.

Kowalewski, K., McLennan, J. D., \& McGrath, P. J. (2011). A preliminary investigation of wait times for child and adolescent mental health services in Canada. Journal of the Canadian Academy of Child and Adolescent Psychiatry, 20, 112-119.

Lewin, G. W. (1944). Constructs in field theory. Resolving social conflicts and field theory in social science (pp. 191-199). New York: Harper and Brothers.

Loughman, A., \& Haslam, N. (2018). Neuroscientific explanations and the stigma of mental disorder: a meta-analytic study. Cognitive Research: Principles and Implications, 3(1), 43.

Lutz, W., Schwartz, B., Hofmann, S., Fisher, A., Husen, K., \& Rubel, J. (2018). Using network analysis for the prediction of treatment dropout in patients with mood and anxiety disorders: A methodological proof-of-concept study. Scientific Reports.

MacDuffie, K. E., \& Strauman, T. J. (2017). Understanding Our Own Biology: The Relevance of AutoBiological Attributions for Mental Health. Clinical Psychology: Science and Practice, 24, 50-68.

Makridakis, S., Spiliotis, E., \& Assimakopoulos, V. (2018). Statistical and Machine Learning forecasting methods: Concerns and ways forward. PloS One, 13(3), e0194889.

Malowney, M., Keltz, S., Fischer, D., \& Boyd, J. W. (2015). Availability of outpatient care from psychiatrists: a simulated-patient study in three U.S. cities. Psychiatric Services , 66(1), 94-96.

Mendenhall, E., De Silva, M. J., Hanlon, C., Petersen, I., Shidhaye, R., Jordans, M., ... Lund, C. (2014). Acceptability and feasibility of using non-specialist health workers to deliver mental health care: Stakeholder perceptions from the PRIME district sites in Ethiopia, India, Nepal, South Africa, and Uganda. Social Science \& Medicine, 118, 33-42.

Miu, A. S., \& Yeager, D. S. (2015). Preventing symptoms of depression by teaching adolescents that people can change effects of a brief incremental theory of personality intervention at 9-month follow-up. Clinical Psychological Science, 3(5), 726-743.

Mojtabai, R., Olfson, M., \& Han, B. (2016). National Trends in the Prevalence and Treatment of Depression in Adolescents and Young Adults. Pediatrics, 138. 
Moshontz, H., Campbell, L., Ebersole, C. R., IJzerman, H., Urry, H. L., Forscher, P. S., ... Chartier, C. R. (2018). The Psychological Science Accelerator: Advancing Psychology Through a Distributed Collaborative Network. Advances in Methods and Practices in Psychological Science, 1, 501-515.

Muñoz, R. F., Bunge, E. L., Chen, K., Schueller, S. M., Bravin, J. I., Shaughnessy, E. A., \& Pérez-Stable, E. J. (2016). Massive Open Online Interventions. Clinical Psychological Science, 4, 194-205.

Nieuwsma, J. A., Trivedi, R. B., McDuffie, J., Kronish, I., Benjamin, D., \& Williams, J. W. (2012). Brief psychotherapy for depression: a systematic review and meta-analysis. International Journal of Psychiatry in Medicine, 43(2), 129-151.

Ollendick, T. H., Ryan, S. M., Capriola-Hall, N. N., Austin, K. E., \& Fraire, M. (2018). Have Phobias, Will Travel: Addressing One Barrier to the Delivery of an Evidence-Based Treatment. Behavior Therapy, 49(4), 594-603.

Öst, L.-G., \& Ollendick, T. H. (2017). Brief, intensive and concentrated cognitive behavioral treatments for anxiety disorders in children: A systematic review and meta-analysis. Behaviour Research and Therapy, 97, 134-145.

Piccirillo, M. L., \& Rodebaugh, T. L. (2019, in press). Foundations of idiographic methods in psychology and applications for psychotherapy. Clinical Psychology Review.

Ranney, M. L., Goldstick, J., Eisman, A., Carter, P. M., Walton, M., \& Cunningham, R. M. (2017). Effects of a brief ED-based alcohol and violence intervention on depressive symptoms. General Hospital Psychiatry, 46, 44-48.

Roehrig, C. (2016). Mental Disorders Top The List Of The Most Costly Conditions In The United States: \$201 Billion. Health Affairs, 35(6), 1130-1135.

Rothì, D. M., Leavey, G., \& Best, R. (2008). On the front-line: Teachers as active observers of pupils' mental health. Teaching and Teacher Education, 24, 1217-1231.

Ruscio, J., \& Mullen, T. (2012). Confidence intervals for the probability of superiority effect size measure and the area under a receiver operating characteristic curve. Multivariate Behavioral Research, 47(2), 201-223. 
Samdal, O., \& Rowling, L. (2015). Implementation Strategies to Promote and Sustain Health and Learning in School. Schools for Health and Sustainability, 233-252.

Sanders, M. R., \& Turner, K. M. T. (2005). Reflections on the challenges of effective dissemination of behavioural family intervention: Our experience with the Triple P--Positive Parenting Program. Child and Adolescent Mental Health, 10(4), 158-169.

Schleider, J. L., Abel, M. R., \& Weisz, J. R. (2019). Do Immediate Gains Predict Long-Term Symptom Change? Findings from a Randomized Trial of a Single-Session Intervention for Youth Anxiety and Depression. Child Psychiatry and Human Development.

Schleider, J. L., Burnette, J. L., Widman, L., Hoyt, C., \& Prinstein, M. J. (2019). Randomized Trial of a Single-Session Growth Mind-Set Intervention for Rural Adolescents' Internalizing and Externalizing Problems. Journal of Clinical Child \& Adolescent Psychology.

Schleider, J. L., Dobias, M. L., \& Pati, S. (2019, pre-print). Promoting treatment access following pediatric primary care depression screening: Randomized trial of web-based, single-session interventions for parents and youths. OSF Preprints. doi.org/10.31219/osf.io/c639z

Schleider, J. L., Mullarkey, M. C., Mumper, E., \& Sung, J. Y. (2019). The A.B.C. Porject. Open Science Framework. doi.org/10.17605/OSF.IO/QJ94C

Schleider, J. L., Mullarkey, M. C., \& Chacko, A. (2019). Harnessing Wise Interventions to Advance the Potency and Reach of Youth Mental Health Services. Clinical Child and Family Psychology Review.

Schleider, J. L., Mullarkey, M. C., \& Weisz, J. R. (2019). Virtual Reality and Web-Based Growth Mindset Interventions for Adolescent Depression: Protocol for a Three-Arm Randomized Trial. JMIR Research Protocols, 8(7), e13368.

Schleider, J. L., \& Weisz, J. R. (2019). Project Personality. Open Science Framework. DOI 10.17605/OSF.IO/259JV

Schleider, J. L., \& Weisz, J. R. (2019). Sharing Feelings Project. Open Science Framework. DOI 10.17605/OSF.IO/SWV5B

Schleider, J. L., \& Weisz, J. R. (2016). Reducing risk for anxiety and depression in adolescents: Effects of 
a single-session intervention teaching that personality can change. Behaviour Research and Therapy, 87, 170-181.

Schleider, J. L., \& Weisz, J. R. (2017). Little Treatments, Promising Effects? Meta-Analysis of SingleSession Interventions for Youth Psychiatric Problems. Journal of the American Academy of Child \& Adolescent Psychiatry, Vol. 56, pp. 107-115. https://doi.org/10.1016/j.jaac.2016.11.007

Schleider, J. L., \& Weisz, J. R. (2018). Parent Expectancies and Preferences for Mental Health Treatment: The Roles of Emotion Mind-Sets and Views of Failure. Journal of Clinical Child and Adolescent Psychology: The Official Journal for the Society of Clinical Child and Adolescent Psychology, American Psychological Association, Division 53, 47(sup1), S480-S496.

Schleider, J., \& Weisz, J. (2018). A single-session growth mindset intervention for adolescent anxiety and depression: 9-month outcomes of a randomized trial. Journal of Child Psychology and Psychiatry, and Allied Disciplines, 59(2), 160-170.

Shen, F., Sheer, V. C., \& Li, R. (2015). Impact of Narratives on Persuasion in Health Communication: A Meta-Analysis. Journal of Advertising, Vol. 44, pp. 105-113. https://doi.org/10.1080/00913367.2015.1018467

Stiffman, A. R., Pescosolido, B., \& Cabassa, L. J. (2004). Building a model to understand youth service access: the gateway provider model. Mental Health Services Research, 6(4), 189-198.

Strauss, M. E., \& Smith, G. T. (2009). Construct validity: advances in theory and methodology. Annual Review of Clinical Psychology, 5, 1-25.

Talmon, M. (1990). Single Session Therapy: Maximizing the Effect of the First (and Often Only) Therapeutic Encounter. Jossey-Bass.

Tingley, D., Yamamoto, T., Hirose, K., Keele, L., \& Imai, K. (2015). mediation: Causal Mediation Analysis (Version 4.4.5).

United Nations. General Assembly. (1989). Convention on the Rights of the Child, 20 November 1989. Annual Review of Population Law, 16, 95, 485-501.

van Laer, T., Feiereisen, S., \& Visconti, L. M. (2019). Storytelling in the digital era: A meta-analysis of 
relevant moderators of the narrative transportation effect. Journal of Business Research, 96, 135146. https://doi.org/10.1016/j.jbusres.2018.10.053

Wallerstein, N., \& Duran, B. (2010). Community-based participatory research contributions to intervention research: the intersection of science and practice to improve health equity. American Journal of Public Health, 100 Suppl 1, S40-S46.

Walsh, C. G., Ribeiro, J. D., \& Franklin, J. C. (2017). Predicting risk of suicide attempts over time through machine learning. Clinical Psychological Science, 5(3), 457-469.

White, C., Shanley, D. C., Zimmer-Gembeck, M. J., Walsh, K., Hawkins, R., \& Lines, K. (2019). Outcomes of In Situ Training for Disclosure as a Standalone and a Booster to a Child Protective Behaviors Education Program. Child Maltreatment, 24, 193-202.

Yarkoni, T., \& Westfall, J. (2017). Choosing Prediction Over Explanation in Psychology: Lessons From Machine Learning. Perspectives on Psychological Science: A Journal of the Association for Psychological Science, 12(6), 1100-1122.

Yeager, D. S., \& Walton, G. M. (2011). Social-Psychological Interventions in Education. Review of Educational Research, Vol. 81, pp. 267-301. https://doi.org/10.3102/0034654311405999

Zou, H., \& Hastie, T. (2005). Regularization and variable selection via the elastic net. Journal of the Royal Statistical Society. Series B, Statistical Methodology, 67(2), 301-320. 
1 a.

What we do (our actions) can change how we feel - for better and for worse This includes how we act after going through stress and setbacks. Take 16-year-old Kat's story as one example.

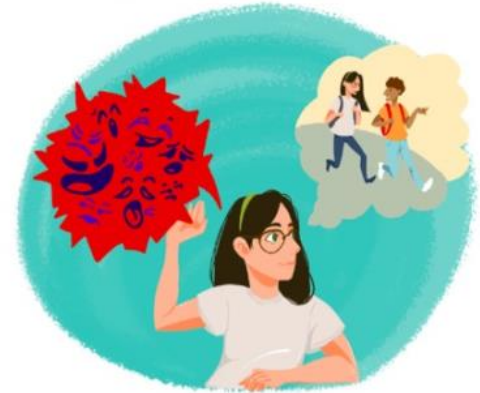

"I've played volleyball since I was 8 years old. It's always been my favorite thing. Last year, I decided to try out for my school's varsity team. My best friend made it, but I got cut. I felt sick when I found out. My friend was nice about it, but I was just so mad at myself. I felt stupid for thinking I could make it. After that, things got tough. I just wanted to hide. Hanging out with my friend, being at school-it all seemed like too much. I didn't have energy for anything. Even volleyball stopped being fun. I felt really low during that time. At the time, I didn't understand what was happening.

I remember thinking, "will I ever feel like myself again?"

$1 b$.

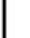

